IUHET-457

August 2003

\title{
Superfield Realizations of Lorentz and CPT Violation
}

\author{
M. S. Berger * \\ Physics Department, Indiana University, Bloomington, IN 47405, USA
}

\begin{abstract}
Superfield realizations of Lorentz-violating extensions of the Wess-Zumino model are presented. These models retain supersymmetry but include terms that explicitly break the Lorentz symmetry. The models can be understood as arising from superspace transformations that are modifications of the familiar one in the Lorentz-symmetric case.
\end{abstract}

*Electronic address: berger@indiana.edu 


\section{INTRODUCTION}

Spacetime symmetries have played an important role in formulation of theories of fundamental physics for the last hundred years. The special and general theories of relativity are founded on underlying spacetime symmetries, and the most popular speculations about the possible advances in physics involve further spacetime symmetries such as supersymmetry [1]. If supersymmetry does in fact describe nature, then it is clear from experimental observation that this is a symmetry that must be broken.

Since spacetime symmetries are central to fundamental particle physics, it is important to consider the consequences of all possible ways of breaking them. Broken supersymmetry has been extensively studied because of the experimental necessity of splitting the masses of the observed particles from their supersymmetric partners. The Lorentz symmetry requires that their be no preferred direction in space and no preferred frame. Whether this symmetry is exact or broken is a question for experiment.

It is usually considered desirable for a broken symmetry to arise spontaneously since then certain properties of the theory that result from the underlying symmetry are retained. Furthermore, it is expected that any symmetry that is approximately valid must have some fundamental realization, and any breaking should arise spontaneously. Presumably the explicit supersymmetry breaking terms that are added to the models used in phenomenological supersymmetric theories such as the Minimal Supersymmetric Standard Model arise in a more fundamental theory from some spontaneous breaking of supersymmetry. Nevertheless, these low-energy supersymmetric theories can be viewed as effective theories.

If one believes in electroweak-scale supersymmetry, one has to accept that a spacetime symmetry is broken. From the point of view of available experiment, this breaking seems to be a very large effect; in fact, the supersymmetry breaking is so large that no superpartners have even been found. However, compared to the fundamental Planck scale, the supersymmetry breaking scale is very small, and supersymmetry is more appropriately viewed as an approximate symmetry. Indeed major effort has gone into trying to understand why the breaking of supersymmetry is so small compared to the Planck scale.

Recent articles [2-4] have studied the possibility of introducing Lorentz-violation into supersymmetric theories. Most theories incorporate the Poincaré symmetry at the outset. This assumption is well-justified on the basis that no violations have ever been observed experimentally. However, in light of the argument just made that supersymmetry is a approximate spacetime symmetry, it seems appropriate to consider the possibility that there is violation of the other spacetime symmetries at some level. Supersymmetry can be broken within the context of local field theory. Lorentz and CPT violation might arise from nonlocal interactions in a more fundamental theory. One approach is to use a field theory treatment of Lorentz and CPT-violation that incorporates their effects by adding explicit terms to a symmetric Lagrangian and the resulting field theories should be regarded as effective theories only. Problems with microcausality are addressed in the underlying fundamental theory at the energy scales at which the effective theory breaks down [5]. The experimental implications of Lorentz and CPT-violation have been explored extensively in recent years [6].

In Ref. [2] we examined the possibility that one could construct a Lagrangian that respects a supersymmetry algebra, but that has terms that explicitly violate the Lorentz symmetry.

Since these models contain Lorentz-violation, they fall outside the usual classification of 
supersymmetry algebras [7]. The conventional supersymmetry algebra is given by a transformation (involving a two-component Weyl spinor $Q$ ) between bosons and fermions that upon anticommutation yields the translation operator:

$$
\begin{aligned}
& {\left[Q, P_{\mu}\right]=0} \\
& \{Q, \bar{Q}\}=2 \sigma^{\mu} P_{\mu} .
\end{aligned}
$$

It was shown that there are indeed simple extensions of the Wess-Zumino model [8] that respect a similar algebra, but that have extra terms that explicitly violate the Lorentz symmetry characterized by the generators of boosts and rotations, $M_{\mu \nu}$,

$$
\begin{aligned}
{\left[P_{\mu}, P_{\nu}\right] } & =0 \\
{\left[P_{\mu}, M_{\rho \sigma}\right] } & =i\left(\eta_{\mu \rho} P_{\sigma}-\eta_{\mu \sigma} P_{\rho}\right) \\
{\left[M_{\mu \nu}, M_{\rho \sigma}\right] } & =i\left(\eta_{\nu \rho} M_{\mu \sigma}-\eta_{\nu \sigma} M_{\mu \rho}-\eta_{\mu \rho} M_{\nu \sigma}+\eta_{\mu \sigma} M_{\nu \rho}\right),
\end{aligned}
$$

The commutation relations in Eqn. (2) form the Poincaré algebra, and when the supersymmetric generators are included as in Eqn. (1), the result is the superPoincaré algebra. Lorentz-violating models will not respect all the commutation relations involving $M_{\mu \nu}$.

Two extensions to the supersymmetric Wess-Zumino model were presented which contain explicit terms contain the Lorentz-violation. One of these models preserves CPT whereas the other is CPT-violating. A superspace formulation for the CPT preserving theory was already presented in Ref. [2]. In this paper we extend the superspace formulation to the CPT-violating model. We show that the Wess-Zumino model and its two extensions all admit a description in terms of transformations on superspace. In addition, we present the two extended models in an alternative form involving two-component Weyl spinors as opposed to the four-component Majorana spinors used in Ref. [2].

The usual Wess-Zumino Lagrangian is elegantly derived in the framework of superspace [9]. A superfield $\Phi(x, \theta, \bar{\theta})$ is a function of the commuting spacetime coordinates $x^{\mu}$ and of four anticommuting coordinates $\theta^{\alpha}$ and $\bar{\theta}_{\dot{\alpha}}$ which form two-component Weyl spinors. A chiral superfield is a function of $y^{\mu}=x^{\mu}+i \theta \sigma^{\mu} \bar{\theta}$ and $\theta$, i.e.

$$
\begin{aligned}
\Phi(x, \theta, \bar{\theta})= & \phi(y)+\sqrt{2} \theta \psi(y)+(\theta \theta) \mathcal{F}(y), \\
= & \phi(x)+i \theta \sigma^{\mu} \bar{\theta} \partial_{\mu} \phi(x)-\frac{1}{4}(\theta \theta)(\bar{\theta} \bar{\theta}) \square \phi(x) \\
& +\sqrt{2} \theta \psi(x)+i \sqrt{2} \theta \sigma^{\mu} \bar{\theta} \theta \partial_{\mu} \psi(x)+(\theta \theta) \mathcal{F}(x)
\end{aligned}
$$

where one can define the usual real components of the complex scalar components as

$$
\phi=\frac{1}{\sqrt{2}}(A+i B), \quad \mathcal{F}=\frac{1}{\sqrt{2}}(F-i G) .
$$

The conjugate superfield is

$$
\begin{aligned}
\Phi^{*}(x, \theta, \bar{\theta})= & \phi^{*}(z)+\sqrt{2} \bar{\theta} \bar{\psi}(z)+(\bar{\theta} \bar{\theta}) \mathcal{F}^{*}(z) \\
= & \phi^{*}(x)-i \theta \sigma^{\mu} \bar{\theta} \partial_{\mu} \phi^{*}(x)-\frac{1}{4}(\theta \theta)(\bar{\theta} \bar{\theta}) \square \phi^{*}(x) \\
& +\sqrt{2} \bar{\theta} \bar{\psi}(x)+i \sqrt{2} \theta \sigma^{\mu} \bar{\theta} \bar{\theta} \partial_{\mu} \bar{\psi}(x)+(\bar{\theta} \bar{\theta}) \mathcal{F}^{*}(x)
\end{aligned}
$$


where $z^{\mu}=x^{\mu}-i \theta \sigma^{\mu} \bar{\theta}=y^{\mu *}$. We have taken the opportunity to reexpress the superfields in terms of Weyl spinors as opposed to the use of Majorana spinors in Ref. [2].

The Lagrangian can be derived from the superspace integral

$$
\int d^{4} \theta \Phi^{*} \Phi+\int d^{2} \theta\left[\frac{1}{2} m \Phi^{2}+\frac{1}{3} g \Phi^{3}+\text { h.c. }\right]
$$

where the superspace integrals elegantly project out the $(\theta \theta)(\bar{\theta} \bar{\theta})$ component of the $\Phi^{*} \Phi$ superfield in the first term, and the $\theta \theta$ component in the second term. The result is the Wess-Zumino Lagrangian,

$$
\begin{aligned}
\mathcal{L}= & \partial_{\mu} \phi^{*} \partial^{\mu} \phi+\frac{i}{2}\left[\left(\partial_{\mu} \psi\right) \sigma^{\mu} \bar{\psi}+\left(\partial_{\mu} \bar{\psi}\right) \bar{\sigma}^{\mu} \psi\right]+\mathcal{F}^{*} \mathcal{F} \\
& +m\left[\phi \mathcal{F}+\phi^{*} \mathcal{F}^{*}-\frac{1}{2} \psi \psi-\frac{1}{2} \bar{\psi} \bar{\psi}\right] \\
& +g\left[\phi^{2} \mathcal{F}+\phi^{* 2} \mathcal{F}^{*}-\phi(\psi \psi)-\phi^{*}(\bar{\psi} \bar{\psi})\right] .
\end{aligned}
$$

The action $\delta_{S} \Phi(x, \theta, \bar{\theta})=-i(\epsilon Q+\bar{\epsilon} \bar{Q}) \Phi(x, \theta, \bar{\theta})$ of the supersymmetry generators $Q$ and $\bar{Q}$

$$
\delta_{S} \Phi(x, \theta, \bar{\theta})=\left[\epsilon^{\alpha} \partial_{\alpha}+\bar{\epsilon}_{\dot{\alpha}} \bar{\partial}^{\dot{\alpha}}+i \theta \sigma^{\mu} \bar{\epsilon} \partial_{\mu}-i \epsilon \sigma^{\mu} \bar{\theta} \partial_{\mu}\right] \Phi(x, \theta, \bar{\theta})
$$

transforms the Lagrangian into itself plus a total derivative.

As shown in Ref. [2], Lorentz-violation can be introduced into the Wess-Zumino Lagrangian via the substitution $\partial_{\mu} \rightarrow \partial_{\mu}+k_{\mu \nu} \partial^{\nu}$,

$$
\begin{aligned}
\mathcal{L}_{\text {Lorentz }}= & \left(\partial_{\mu}+k_{\mu \nu} \partial^{\nu}\right) \phi^{*}\left(\partial^{\mu}+k^{\mu \rho} \partial_{\rho}\right) \phi \\
& +\frac{i}{2}\left[\left(\left(\partial_{\mu}+k_{\mu \nu} \partial^{\nu}\right) \psi\right) \sigma^{\mu} \bar{\psi}+\left(\left(\partial_{\mu}+k_{\mu \nu} \partial^{\nu}\right) \bar{\psi}\right) \bar{\sigma}^{\mu} \psi\right]+\mathcal{F}^{*} \mathcal{F} \\
& +m\left[\phi \mathcal{F}+\phi^{*} \mathcal{F}^{*}-\frac{1}{2} \psi \psi-\frac{1}{2} \bar{\psi} \bar{\psi}\right] \\
& +g\left[\phi^{2} \mathcal{F}+\phi^{* 2} \mathcal{F}^{*}-\phi(\psi \psi)-\phi^{*}(\bar{\psi} \bar{\psi})\right] .
\end{aligned}
$$

In this equation, $k_{\mu \nu}$ is a real, symmetric, traceless, and dimensionless coefficient determining the magnitude of Lorentz-violation. The coefficient $k_{\mu \nu}$ transforms as a 2-tensor under observer Lorentz transformations but as a scalar under particle Lorentz transformations $[10,11]$.

An extension of the Wess-Zumino model that violates CPT in addition to containing Lorentz-violation was introduced in Ref. [2]. The Lagrangian for the model is

$$
\mathcal{L}_{\mathrm{CPT}}=\left[\left(\partial_{\mu}-i k_{\mu}\right) \phi^{*}\right]\left[\left(\partial^{\mu}+i k^{\mu}\right) \phi\right]+\frac{i}{2}\left[\left(\left(\partial_{\mu}+i k_{\mu}\right) \psi\right) \sigma^{\mu} \bar{\psi}+\left(\left(\partial_{\mu}-i k_{\mu}\right) \bar{\psi}\right) \bar{\sigma}^{\mu} \psi\right]+\mathcal{F}^{*} \mathcal{F} .
$$

Here the Lorentz and CPT-violation is controlled by $k_{\mu}$, which is a real coefficient of mass dimension one transform as a four-vector under observer Lorentz transformations but is unaffected by particle Lorentz transformations $[10,11]$. Unlike the coefficient $k_{\mu \nu}$, the quantity $k_{\mu}$ as an odd number of four-indices so it violates CPT. It has been shown on quite general grounds that CPT-violation implies Lorentz violation [12,13]. The Lagrangian for the model with the CPT-violating coefficient $k_{\mu}$ can be obtained from the kinetic part of the Wess-Zumino Lagrangian in Eqn. (7) with the appropriate substitutions $\partial_{\mu} \rightarrow \partial_{\mu} \pm i k_{\mu}$. 


\section{MODIFIED SUPERFIELDS}

The two Lorentz-violating models can be understood in the superspace formalism in a way that parallels that of the ordinary Wess-Zumino model. Define superfields

$$
\begin{aligned}
\Phi_{y}(x, \theta, \bar{\theta})= & \Phi\left(x, \theta, \bar{\theta} ; \partial_{\mu} \rightarrow \partial_{\mu}+k_{\mu \nu} \partial^{\nu}\right) \\
= & \phi\left(x_{+}\right)+\sqrt{2} \theta \psi\left(x_{+}\right)+(\theta \theta) F\left(x_{+}\right) \\
= & \phi(x)+i \theta \sigma^{\mu} \bar{\theta}\left(\partial_{\mu}+k_{\mu \nu} \partial^{\nu}\right) \phi(x)-\frac{1}{4}(\theta \theta)(\bar{\theta} \bar{\theta})\left(\partial_{\mu}+k_{\mu \nu} \partial^{\nu}\right)\left(\partial^{\mu}+k^{\mu \rho} \partial_{\rho}\right) \phi(x) \\
& +\sqrt{2} \theta \psi(x)+i \sqrt{2} \theta \sigma^{\mu} \bar{\theta} \theta\left(\partial_{\mu}+k_{\mu \nu} \partial^{\nu}\right) \psi(x)+(\theta \theta) F(x),
\end{aligned}
$$

and

$$
\begin{aligned}
\Phi_{y}^{*}(x, \theta, \bar{\theta})= & \Phi^{*}\left(x, \theta, \bar{\theta} ; \partial_{\mu} \rightarrow \partial_{\mu}+k_{\mu \nu} \partial^{\nu}\right) \\
= & \phi^{*}\left(x_{-}\right)+\sqrt{2} \bar{\theta} \bar{\psi}\left(x_{-}\right)+(\bar{\theta} \bar{\theta}) F^{*}\left(x_{-}\right) \\
= & \phi^{*}(x)-i \theta \sigma^{\mu} \bar{\theta}\left(\partial_{\mu}+k_{\mu \nu} \partial^{\nu}\right) \phi^{*}(x)-\frac{1}{4}(\theta \theta)(\bar{\theta} \bar{\theta})\left(\partial_{\mu}+k_{\mu \nu} \partial^{\nu}\right)\left(\partial^{\mu}+k^{\mu \rho} \partial_{\rho}\right) \phi^{*}(x) \\
& +\sqrt{2} \bar{\theta} \bar{\psi}(x)+i \sqrt{2} \theta \sigma^{\mu} \bar{\theta} \bar{\theta}\left(\partial_{\mu}+k_{\mu \nu} \partial^{\nu}\right) \bar{\psi}(x)+(\bar{\theta} \bar{\theta}) F^{*}(x)
\end{aligned}
$$

where

$$
x_{ \pm}^{\mu}=x^{\mu} \pm i \theta \sigma^{\mu} \bar{\theta} \pm i k^{\mu \nu} \theta \sigma_{\nu} \bar{\theta},
$$

are shifted coordinates that take the place of $y^{\mu}$ and $z^{\mu}$. Under a CPT-transformation the chiral superfield $\Phi_{y}$ and the antichiral superfield $\Phi_{y}^{*}$ transform into themselves just as the usual superfields $\Phi$ and $\Phi^{*}$ do. The Lagrangian in Eqn. (9) can be obtained by the same superspace integral in Eqn. (6) with the superfields $\Phi_{y}$ and $\Phi_{y}^{*}$ substituted in the place of $\Phi$ and $\Phi^{*}$ (see Eqn. (29) below).

As argued in Ref. [2], it is clear that the Lagrangian in Eqn. (9) transforms into a total derivative under the supersymmetric transformation

$$
\delta_{S} \Phi_{y}(x, \theta, \bar{\theta})=\left[\epsilon^{\alpha} \partial_{\alpha}+\bar{\epsilon}_{\dot{\alpha}} \bar{\partial}^{\dot{\alpha}}+i \theta \sigma^{\mu} \bar{\epsilon}\left(\partial_{\mu}+k_{\mu \nu} \partial^{\nu}\right)-i \epsilon \sigma^{\mu} \bar{\theta}\left(\partial_{\mu}+k_{\mu \nu} \partial^{\nu}\right)\right] \Phi_{y}(x, \theta, \bar{\theta})
$$

since it is simply the usual supersymmetric transformation in Eqn. (1) with the substitution $\partial_{\mu} \rightarrow \partial_{\mu}+k_{\mu \nu} \partial^{\nu}$. The superalgebra generated by $Q$ and $P_{\mu}=i \partial_{\mu}$ is

$$
\left[P_{\mu}, Q\right]=0, \quad\{Q, \bar{Q}\}=2 \sigma^{\mu} P_{\mu}+2 k_{\mu \nu} \sigma^{\mu} P^{\nu} .
$$

Consider now the CPT-violating model in Eqn (10). Define modified superfields by the substitutions

$$
\begin{aligned}
\Phi_{k}(x, \theta, \bar{\theta})= & \Phi\left(x, \theta, \bar{\theta} ; \partial_{\mu} \rightarrow \partial_{\mu}+i k_{\mu}\right) \\
= & \phi(x)+\theta \sigma^{\mu} \bar{\theta}\left(i \partial_{\mu}-k_{\mu}\right) \phi(x)+\frac{1}{4}(\theta \theta)(\bar{\theta} \bar{\theta})\left(i \partial_{\mu}-k_{\mu}\right)\left(i \partial^{\mu}-k^{\mu}\right) \phi(x) \\
& +\sqrt{2} \theta \psi(x)+\sqrt{2} \theta \sigma^{\mu} \bar{\theta} \theta\left(i \partial^{\mu}-k^{\mu}\right) \psi(x)+(\theta \theta) F(x),
\end{aligned}
$$


and

$$
\begin{aligned}
\Phi_{k}^{*}(x, \theta, \bar{\theta})= & \Phi^{*}\left(x, \theta, \bar{\theta} ; \partial_{\mu} \rightarrow \partial_{\mu}-i k_{\mu}\right) \\
= & \phi^{*}(x)-\theta \sigma^{\mu} \bar{\theta}\left(i \partial_{\mu}+k_{\mu}\right) \phi^{*}(x)+\frac{1}{4}(\theta \theta)(\bar{\theta} \bar{\theta})\left(i \partial_{\mu}+k_{\mu}\right)\left(i \partial^{\mu}+k^{\mu}\right) \phi^{*}(x) \\
& +\sqrt{2} \bar{\theta} \bar{\psi}(x)+\sqrt{2} \theta \sigma^{\mu} \bar{\theta} \bar{\theta}\left(i \partial_{\mu}+k_{\mu}\right) \bar{\psi}(x)+(\bar{\theta} \bar{\theta}) F^{*}(x) .
\end{aligned}
$$

The infinitesimal supersymmetry transformations acting on a superfield $\mathcal{S}$

$$
\begin{aligned}
\delta_{S} \Phi_{k}(x, \theta, \bar{\theta}) & =\left[\epsilon^{\alpha} \partial_{\alpha}+\bar{\epsilon}_{\dot{\alpha}} \bar{\partial}^{\dot{\alpha}}+i \theta \sigma^{\mu} \bar{\epsilon}\left(\partial_{\mu}+i k_{\mu}\right)-i \epsilon \sigma^{\mu} \bar{\theta}\left(\partial_{\mu}-i k_{\mu}\right)\right] \Phi_{k}(x, \theta, \bar{\theta}) \\
& =\left[\epsilon^{\alpha}\left(\partial+k_{\mu} \sigma^{\mu} \bar{\theta}\right)_{\alpha}+\bar{\epsilon}_{\dot{\alpha}}\left(\partial-k_{\mu} \theta \sigma^{\mu}\right)^{\dot{\alpha}}+i \theta \sigma^{\mu} \bar{\epsilon} \partial_{\mu}-i \epsilon \sigma^{\mu} \bar{\theta} \partial_{\mu}\right] \Phi_{k}(x, \theta, \bar{\theta})
\end{aligned}
$$

closes by construction on real superfields, $\mathcal{S}^{*}=\mathcal{S}$. However, it does not close on the superfields $\Phi_{k}$ and $\Phi_{k}^{*}$ as can be explicitly checked by a short calculation ${ }^{1}$. The obstruction to defining a supersymmetry generator on chiral superfields was already pointed out in Ref. [2]. The objects $\Phi_{k}$ and $\Phi_{k}^{*}$ are conjugates and can be used to construct real superfields. Since the supersymmetric transformation in Eqn. (18) closes on all real superfields, it closes on $\Phi_{k}^{*} \Phi_{k}$.

\section{SUPERSPACE TRANSFORMATIONS}

Now we proceed to show how the Lorentz-violating extensions of the Wess-Zumino model can be understood as transformations on the superfields. Define

$$
\begin{aligned}
& X \equiv\left(\theta \sigma^{\mu} \bar{\theta}\right) \partial_{\mu}, \\
& Y \equiv k_{\mu \nu}\left(\theta \sigma^{\mu} \bar{\theta}\right) \partial^{\nu}, \\
& K \equiv k_{\mu}\left(\theta \sigma^{\mu} \bar{\theta}\right),
\end{aligned}
$$

so that

$$
\begin{aligned}
& U_{x} \equiv e^{i X}=1+i\left(\theta \sigma^{\mu} \bar{\theta}\right) \partial_{\mu}-\frac{1}{4}(\theta \theta)(\bar{\theta} \bar{\theta}) \square \\
& U_{y} \equiv e^{i Y}=1+i k_{\mu \nu}\left(\theta \sigma^{\mu} \bar{\theta}\right) \partial^{\nu}-\frac{1}{4} k_{\mu \nu} k^{\mu \rho}(\theta \theta)(\bar{\theta} \bar{\theta}) \partial^{\nu} \partial_{\rho}, \\
& T_{k} \equiv e^{-K}=1-k_{\mu}\left(\theta \sigma^{\mu} \bar{\theta}\right)+\frac{k^{2}}{4}(\theta \theta)(\bar{\theta} \bar{\theta}) .
\end{aligned}
$$

Since $X$ and $Y$ are derivative operators, the action of $U_{x}$ and $U_{y}$ on a superfield $\mathcal{S}$ can be understood as a coordinate shift. In the customary (Lorentz symmetric) case involving $U_{x}$ one has

$$
U_{x} \mathcal{S}(x, \theta, \bar{\theta})=\mathcal{S}(y, \theta, \bar{\theta})
$$

\footnotetext{
${ }^{1}$ Application of the supersymmetric transformation in Eqn. (18) to the chiral superfield $\Phi_{k}$ generates components in the antichiral superfield $\Phi_{k}^{*}$, and vice versa.
} 
i.e. the spacetime coordinate $x^{\mu}$ is shifted to $y^{\mu}$. The chiral superfield $\Phi(x, \theta, \bar{\theta})$ is a function of $y^{\mu}$ and $\theta$ only, so it must then be of the form $\Phi(x, \theta, \bar{\theta})=U_{x} \Psi(x, \theta)$ for some function $\Psi$. The chiral superfield $\Phi(x, \theta, \bar{\theta})$ does not depend on $\bar{\theta}$ except through the coordinate $y^{\mu}$. As is well-known, the kinetic terms of the Wess-Zumino model can be expressed as

$$
\int d^{4} \theta\left[U_{x}^{*} \Psi(x, \bar{\theta})^{*}\right]\left[U_{x} \Psi(x, \theta)\right]=\int d^{4} \theta \Phi^{*}(z, \bar{\theta}) \Phi(y, \theta) .
$$

The supersymmetric models with Lorentz-violating terms can be expressed in terms of superfields in an analogous way. Consider the superfields

$$
\begin{aligned}
\Phi_{y}(x, \theta, \bar{\theta}) & =U_{y} U_{x} \Psi(x, \theta), \\
\Phi_{y}^{*}(x, \theta, \bar{\theta}) & =U_{y}^{*} U_{x}^{*} \Psi^{*}(x, \bar{\theta}) \\
& =U_{y}^{-1} U_{x}^{-1} \Psi^{*}(x, \bar{\theta}) .
\end{aligned}
$$

Applying $U_{y}$ to the chiral and antichiral superfields merely effects the substitution $\partial_{\mu} \rightarrow$ $\partial_{\mu}+k_{\mu \nu} \partial^{\nu}$. Since $U_{y}$ involves a derivative operator just as $U_{x}$, the derivation of the chiral superfield $\Phi_{y}$ can be understood as a function of the variables $x_{+}^{\mu}$ and $\theta$ analogous to how, in the usual case, $\Phi$ is a function of the variables $y^{\mu}$ and $\theta$. The Lagrangian is given by

$$
\begin{aligned}
& \int d^{4} \theta \Phi_{y}^{*} \Phi_{y}+\int d^{2} \theta\left[\frac{1}{2} m \Phi_{y}^{2}+\frac{1}{3} g \Phi_{y}^{3}+\text { h.c. }\right] \\
& =\int d^{4} \theta\left[U_{y}^{*} \Phi^{*}\right]\left[U_{y} \Phi\right]+\int d^{2} \theta\left[\frac{1}{2} m \Phi^{2}+\frac{1}{3} g \Phi^{3}+\text { h.c. }\right] .
\end{aligned}
$$

For the CPT-violating model the superfields have the form

$$
\begin{aligned}
\Phi_{k}(x, \theta, \bar{\theta}) & =T_{k} U_{x} \Psi(x, \theta), \\
\Phi_{k}^{*}(x, \theta, \bar{\theta}) & =T_{k}^{*} U_{x}^{*} \Psi^{*}(x, \bar{\theta}) \\
& =T_{k} U_{x}^{-1} \Psi^{*}(x, \bar{\theta}) .
\end{aligned}
$$

It is helpful to note that the transformation $U_{x}$ acts on $\Psi$ and its inverse $U_{x}^{-1}$ acts on $\Psi^{*}$, while the same transformation $T_{k}$ acts on both $\Psi$ and $\Psi^{*}$ (rather than its inverse). A consequence of this fact is that the supersymmetry transformation will act differently on the components of the chiral superfield and its conjugate as described in Ref. [2]. Specifically the chiral superfield $\Phi_{k}$ is the same as $\Phi$ with the substitution $\partial_{\mu} \rightarrow \partial_{\mu}+i k_{\mu}$ whereas the antichiral superfield $\Phi_{k}^{*}$ is the same as $\Phi^{*}$ with the substitution $\partial_{\mu} \rightarrow \partial_{\mu}-i k_{\mu}$.

The CPT-violating model in Eqn. (10) can then be represented in the following way as a superspace integral:

$$
\int d^{4} \theta \Phi_{k}^{*} \Phi_{k}=\int d^{4} \theta \Phi^{*} e^{-2 K} \Phi
$$

The projection factor $e^{-2 K}$ commutes through the superfields, but its placement in Eqn. (32) 
is suggestive of the coupling of a chiral superfield to a gauge field ${ }^{2}$. Unlike the CPTconserving model, the $(\theta \theta)(\bar{\theta} \bar{\theta})$ component of $\Phi^{*} \Phi$ no longer transforms into a total derivative. A certain combination of components of $\Phi^{*} \Phi$ does transform into a total derivative, and this combination can be understood as being the $(\theta \theta)(\bar{\theta} \bar{\theta})$ component of $\Phi_{k}^{*} \Phi_{k}$. Therefore, we have achieved a superspace formulation of the CPT-violating supersymmetric model that was first described in Ref. [2].

As mentioned above, the supersymmetry transformation does not close for a chiral superfield. The components of the standard chiral supermultiplet $\Phi$ and its conjugate $\Phi^{*}$ each transform into themselves under a CPT-transformation (as opposed to a parity transformation which interchange the components of the supermultiplet with those of its conjugate). On the other hand, for $\Phi_{k}$ and $\Phi_{k}^{*}$, the CPT transformation takes $k_{\mu} \rightarrow-k_{\mu}$, so that the chiral and antichiral superfields $\Phi_{k}$ and $\Phi_{k}^{*}$ mix under it.

\section{CONCLUSIONS}

Extensions of the Wess-Zumino model that contain terms that violate the Lorentz symmetry but preserve the supersymmetric part of the superPoincaré algebra exist. The simplest extension preserves CPT and is obtained by the substitution $\partial_{\mu} \rightarrow \partial_{\mu}+k_{\mu \nu} \partial^{\nu}$. Since this substitution replaces a derivative operator with another derivative operator, the $(\theta \theta)(\bar{\theta} \bar{\theta})$ component of a vector superfield and the $\theta \theta$ component of functions of a chiral superfield still transform as total derivatives. The projection from superfields to components proceeds in the usual way. In fact one can introduce the new coordinate $x_{+}^{\mu}=x^{\mu}+i \theta \sigma^{\mu} \bar{\theta}+i k^{\mu \nu} \theta \sigma_{\nu} \bar{\theta}$ and obtain the chiral superfield as the most general function of this $y$ and $\theta$. It is clear that adding Lorentz-violation in this fashion can be immediately extended to encompass supersymmetric gauge theories as well.

The CPT-violating model, however, does not involve adding a derivative operator. A certain combination of the components of the $\Phi^{*} \Phi$ superfield does in fact transform into a total derivative. This combination can be projected out of the vector superfield by applying the operator $e^{-2 k_{\mu}\left(\theta \sigma^{\mu} \bar{\theta}\right)}$ and then performing the usual projection of the $(\theta \theta)(\bar{\theta} \bar{\theta})$ component. One obtains precisely the CPT-violating model presented in Ref. [2]

The conventional Wess-Zumino model can be described in terms of superspace transformations and projecting out the highest component of the result. It was shown that the two Lorentz-violating models can be understood in terms of similar transformations on the superfields.

${ }^{2}$ The kinetic terms of a supersymmetric gauge theory for which the kinetic terms can be expressed as

$$
\int d^{4} \theta \Phi^{*} e^{2 g V} \Phi
$$

where $V$ is a vector superfield, and the ordinary derivatives $\partial_{\mu}$ are replaced by gauge covariant derivative $D_{\mu}=\partial_{\mu} \pm i g v_{\mu}$ where $g$ is the gauge coupling and $v_{\mu}$ is the component of $V$ multiplying $\theta \sigma^{\mu} \bar{\theta}$. The sign in the covariant derivative is different for the chiral and antichiral superfields. 


\section{ACKNOWLEDGMENTS}

This work was supported in part by the U.S. Department of Energy under Grant No. No. DE-FG02-91ER40661. 


\section{REFERENCES}

[1] For textbooks on the subject, see, for example, S. Weinberg, The Quantum Theory of Fields, Vol. III, Cambridge University Press, Cambridge, 2000; J. Wess and J. Bagger, Supersymmetry and Supergravity, 2nd ed., Princeton University Press, Princeton, 1992; I. L. Buchbinder and S. M. Kuzenko, Ideas And Methods Of Supersymmetry And Supergravity: Or A Walk Through Superspace. Institute of Physics Publishing, London, 1995.

[2] M. S. Berger and V. A. Kostelecky, Phys. Rev. D 65, 091701 (2002) [arXiv:hepth/0112243].

[3] M. S. Berger, Presented at Coral Gables Conference on Short Distance Behavior of Fundamental Interactions, Fort Lauderdale, Florida, 11-14 Dec 2002, arXiv:hep-ph/0212353.

[4] H. Belich, J. L. Boldo, L. P. Colatto, J. A. Helayel-Neto and A. L. Nogueira, arXiv:hepth/0304166.

[5] V. A. Kostelecky and R. Lehnert, Phys. Rev. D 63, 065008 (2001) [arXiv:hepth/0012060].

[6] V. A. Kostelecky, "Proceedings of the Second Meeting on CPT and Lorentz Symmetry Meeting, Bloomington, USA, 15-18 August 2001."

[7] R. Haag, J. T. Lopuszanski and M. Sohnius, Nucl. Phys. B 88, 257 (1975).

[8] J. Wess and B. Zumino, Nucl. Phys. B 70, 39 (1974).

[9] A. Salam and J. Strathdee, Nucl. Phys. B 76, 477 (1974).

[10] D. Colladay and V. A. Kostelecky, Phys. Rev. D 55, 6760 (1997) [arXiv:hep-ph/9703464].

[11] D. Colladay and V. A. Kostelecky, Phys. Rev. D 58, 116002 (1998) [arXiv:hep$\mathrm{ph} / 9809521]$.

[12] O. W. Greenberg, Phys. Rev. Lett. 89, 231602 (2002) [arXiv:hep-ph/0201258].

[13] O. W. Greenberg, arXiv:hep-ph/0305276. 